\title{
Oculomotor evidence of sequence learning on the serial reaction time task
}

\author{
DAVID J. MARCUS, CANAN KARATEKIN, and STEVEN MARKIEWICZ \\ University of Minnesota, Minneapolis, Minnesota
}

\begin{abstract}
Manual and oculomotor measures of sequence learning were examined on the serial reaction time (SRT) task. Participants were assigned into four groups differing on response modality (manual, oculomotor) and trial type (sequence, pseudorandom). The pattern of manual RTs replicated previous studies. Frequency of anticipatory eye movements followed similar patterns as RTs. Participants made many anticipations, even in pseudorandom blocks, and frequency of anticipations did not depend on presence of concurrent manual responses. Excluding participants with explicit awareness did not change results. Anticipations were negatively related to RTs in both incidental and intentional learning. Anticipations were positively related to sequence recall in intentional, but not incidental, learning. Results suggest that (1) anticipatory eye movements reflected sequence learning and (2) participants made overt and covert shifts of visuospatial attention to likely stimulus locations prior to stimulus onset, whether or not they made manual responses and whether or not there was a sequence.
\end{abstract}

Whether or not we are aware of it, many activities in our daily lives require the ability to extract regularities from environmental input and to act on the basis of these internal representations. One aspect of this remarkable ability is learning to extract regularities in sequential stimuli and to execute corresponding sequences of actions.

Sequence learning is often measured on the serial reaction time (SRT) task (Nissen \& Bullemer, 1987). The advantage of this task is that it assesses sequence learning through performance measures during the task, rather than through recall or recognition measures administered later. On this task, series of stimuli are displayed briefly in one of several (typically three or four) locations arranged horizontally on a computer screen. Participants respond by pressing buttons corresponding to the locations. Although participants are not informed of this, the stimuli sometimes follow a repeating sequence (typically, 10 or 12 items in length) presented multiple times across blocks. As the sequences recur, manual RTs decrease. When random trials are later presented, RTs increase. The interpretation of these RT changes is that sequence learning has occurred. Because direct measures typically show that awareness of the sequence is poor, the SRT task has been interpreted as demonstrating a predominantly implicit form of learning.

The SRT task has been very useful in investigating sequence learning in healthy adults and clinical populations. Our goal in using this task was to extend this research

The authors thank Christopher Bingham for his assistance with statistical analyses, especially the modeling of random performance on the sequence awareness tasks. Correspondence should be addressed to D. J. Marcus, who is now at the Pediatric Neuropsychology Program, Children's National Medical Center, 14801 Physicians Lane, Suite 173, Rockville, MD 20850 (e-mail: dmarcus@cnmc.org). to typically developing and psychiatrically impaired children. However, one problem in testing clinical and developmental populations on any task in which manual RT is the main dependent variable is the issue of response speed. When there are differences between age groups or between clinical and healthy groups in speed of musculoskeletal responses, it is difficult to tease apart developmental or clinical differences in sequence learning from differences in motor output (cf. Helmuth, Mayr, \& Daum, 2000).

One way to circumvent this problem is to assess performance using eye movements, which are less affected by developmental and clinical factors. Thus, if eye movements can be shown to reflect sequence learning on the SRT task, then an oculomotor version of the task could be helpful in developmental and clinical studies. Therefore, the primary goal of this study was to develop an oculomotor version of the SRT task by comparing eye movements and manual responses of healthy adult participants who only looked at the stimuli with those of participants who both looked and made manual responses.

However, a review of the studies on the SRT task reveals that the issue of what participants are actually looking at, and what they may be learning, if anything, when they observe a sequence of stimuli is still far from clear. Indeed, although this question is very relevant to current theories of SRT learning, there are no studies of the typical SRT task in which eye movements were recorded. Therefore, a secondary purpose of this study was to use the eye movement data to address certain theoretical issues regarding the nature of sequence learning.

In the next section, we first review several theories of sequence learning on the SRT task and then focus on observational studies. Finally, we will examine studies of anticipatory movements during sequence learning. 


\section{What is Learned on the SRT Task?}

What is learned on the SRT task is still under debate. One set of theories proposes that sequence learning on the SRT task involves forming associations between stimuli prior to the instantiation of the sequence in a particular effector. These theories (stimulus-stimulus, S-S, or perceptual learning) are based to a large extent on evidence showing that participants can learn sequences through observation alone (Heyes \& Foster, 2002; Howard, Mutter, \& Howard, 1992; also see Remillard, 2003). The inference drawn from these studies is that participants must be learning associations between sequences of stimuli and that motor responses are not necessary for learning.

However, other researchers point to studies that have failed to replicate observational learning of sequences (Kelly \& Burton, 2001; Willingham, 1999) and argue that spatial or motor response factors play a more integral role in sequence learning. There are differences among these researchers in the nature of the relationship they posit between the mental representation of the sequence and its instantiation in spatial frames of reference and motor systems and in the extent to which they emphasize different aspects of responses. Some argue that the representation of the sequence is abstract, on the basis of locations to which "successive responses are to be directed, an effector-free spatial description" (Keele, Jennings, Jones, Caulton, \& Cohen, 1995, p. 17), or that it consists of "relatively abstract response goals" (Grafton, Hazeltine, \& Ivry, 1998), whereas Willingham (1999) ties the representation of the sequence more closely to the sequence of locations to which the responses are to be made. He explains that "sequence learning in the SRT task is motoric in that it is coded in a spatial frame that is privileged to motor representations, but it is not motoric in the sense of being specific to an effector" (p. 570).

Others propose that sequence learning on the SRT task involves forming associations between one response and the next (response-response, or R-R, learning; Nattkemper \& Prinz, 1997) or between the response and its effectthat is, the subsequent stimulus (response-stimulus, R-S, or response-effect learning; Ziessler, 1994, 1998; Ziessler \& Nattkemper, 2001). Zirngibl and Koch (2002) propose that although R-S and R-R learning are both important mechanisms of sequence learning, they are nevertheless dissociable. They argue that participants who do not develop explicit awareness learn by forming $\mathrm{R}-\mathrm{S}$ associations, whereas those who do develop awareness form $\mathrm{R}-\mathrm{R}$ associations or gradually switch from an $\mathrm{R}-\mathrm{S}$ to an $\mathrm{R}-\mathrm{R}$ mode of learning.

An important but neglected fact in theories of spatial sequence learning is that eye movements are both overt manifestations of shifts of visuospatial attention and motor acts. There has been some speculation on the role of eye movements in sequence learning (Mayr, 1996; Remillard, 2003; Stadler, 1989; Willingham, 1999), but eye movements have not been controlled for in most spatial sequence learning studies and have not been recorded in any study of the typical SRT task. As a result, we do not know whether or not participants are learning to execute sequences of eye movements at the same time as they are learning manual response sequences. We also do not know when these eye movements occur in relation to the manual responses, which locations the eye movements are directed toward at different points during learning, whether they are simply epiphenomenal (reflecting underlying shifts of visuospatial attention), or whether they play a functional role in learning. Without this knowledge, theories of spatial sequence learning remain incomplete.

\section{Observational Studies of Spatial Sequence Learning}

Observational studies of spatial sequence learning have yielded conflicting findings. The main question addressed in these studies was whether participants would show evidence of sequence learning after simply watching the stimuli. Five observational studies of sequence learning have been published (Heyes \& Foster, 2002; Howard et al., 1992; Kelly \& Burton, 2001; Seger, 1997; Willingham, 1999, Experiment 1). These studies differ in procedural details (e.g., the nature of the observation, sequence length, order of the blocks, amount of manual practice provided to participants), degree of explicit awareness expressed by the participants, the specific blocks that were compared to test for sequence learning, and the size of the effect.

Nevertheless, the results suggest that if the sequence is simple, relatively short or otherwise likely to result in explicit knowledge, or if participants are given even a minimal amount of practice manually responding to the sequence, there is evidence for observational learning. However, if participants with explicit knowledge are eliminated from the analyses, or if the sequence is made more complex (reducing the possibility of explicit awareness), the effect sizes become smaller, and the case for observational learning becomes weaker.

However, the role of eye movements may have been misjudged in some of these studies. For example, Howard et al. (1992) state that they "were interested in whether motor responses are necessary for serial pattern learning or whether perceptual responses alone are sufficient" (p. 1029). The definitions of the terms perceptual responses and perceptual knowledge are not clarified, although the authors appear to be using these terms to refer to shifts of visuospatial attention. The fact that eye movements are motor acts is not taken into account in the research design or interpretation of the results. Thus, the study cannot be taken as evidence of purely perceptual learning, if the term is used to refer to an absence of any motor activity during learning. Willingham (1999) also notes that he could not

be certain that subjects were indeed watching the stimuli, although they report that they were. To be certain of this, one could measure eye movements, but then one might wonder whether what is purportedly perceptual learning is in fact the learning of a sequence of eye movements. (pp. 565-566)

Another significant shortcoming in these studies is that interpretations of what happens during observational 
learning have been based on inferences from manual RTs to sequence and random blocks or to new sequence blocks presented after the observation conditions. None of the studies examined what the participants were actually looking at when they were instructed to observe the sequence, or whether they may have been learning a sequence of eye movements, as suggested by Willingham (1999). Therefore, we now turn to another set of studies that may be relevant to the investigation of eye movements during sequence learning.

\section{Anticipatory Manual Responses and Eye Movements During Sequence Learning}

When discussing eye movements during sequence learning, the first question is what the most appropriate dependent variable is. On the basis of previous studies of the SRT task, eye movements could be viewed as responses. For instance, although Mayr (1996) acknowledges the possibility of anticipatory eye movements, he states that "both orienting of attention (or eye movements) and selection of a motor output can be considered as responses" (p. 360). Remillard (2003) also notes that "the sudden appearance of a stimulus in the visual field, as occurs in the SRT task, automatically captures attention" (p. 583). Thus, an obvious choice would be to test whether RTs of saccades made after the onset of the stimuli covary with manual RTs.

However, during our pilot testing, it quickly became apparent that anticipatory eye movements were ubiquitous on this task. Indeed, it has been hypothesized (Hoffman, Martin, \& Schilling, 2003; Nissen \& Bullemer, 1987; Posner \& Rothbart, 1992; Remillard, 2003; Stadler, 1989) that, as participants learn a sequence, they anticipate the location of the next stimulus and prepare for it by orienting their attention. Anticipatory eye movements can be useful in testing this hypothesis.

It is hard to examine anticipatory responses in SRT studies because the standard SRT task relies on manual responses made after the onset of the stimuli and yields few very fast responses or pretarget buttonpresses that could be classified as correct anticipations. In general, however, manual RTs show that participants who develop explicit awareness of the sequence (or who are instructed to learn the sequence) make more correct anticipatory manual responses than participants who do not (Thomas \& Nelson, 2001; Willingham, Nissen, \& Bullemer, 1989).

Schvaneveldt and Gomez (1998) used probabilistic sequences to minimize explicit awareness and to increase the frequency of anticipatory errors, and they measured the number of probable responses to improbable stimuli as an index of sequence learning. As they state, "error rates on fixed sequences are very low and tend to covary with RT. Errors are informative in the present paradigm, however, because they appear to be an index of how much participants are anticipating the sequence" (p. 182). The results showed that anticipatory errors were more likely in single-task conditions than in dual-task conditions and in participants who performed poorly on the secondary task than in those who performed well. In other words, the lower the attentional load, the more likely anticipatory errors were. The question of whether anticipatory errors may have been reflecting explicit awareness of the sequence was left open.

There are two studies of anticipatory eye movements during sequence learning. In one study, Miyashita, Rand, Miyachi, and Hikosaka (1996) examined anticipatory saccades in two monkeys as they learned to perform the $2 \times 5$ task, a variant of the SRT task in which the goal is to learn, through trial and error, a five-step sequence in which each step consists of learning to press two buttons on a board in the correct order. The researchers report that the monkeys made saccades prior to the target throughout training. With extensive training, these saccades came to correctly anticipate the targets in a sequence-specific manner. The increase in the frequency of correct anticipatory saccades paralleled an increase in the frequency of anticipatory manual responses.

In a study by Kawashima et al. (1998), healthy adults memorized the order and locations of stimuli that appeared in a seven-item sequence in one of 25 locations, and their eye movements were recorded as they learned the sequence. A control group was presented with stimuli occurring in random locations. The main dependent variable in terms of eye movements was the time to initiate the saccade in relation to the target's appearance. Average saccadic RTs decreased rapidly during the first few cycles through the sequence (dropping from over $200 \mathrm{msec}$ to $0 \mathrm{msec}$ by the 3 rd cycle), and continued to decrease until the 12 th cycle. By the 12 th cycle, RTs had plateaued to $-500 \mathrm{msec}$. Consistent with Miyashita et al. (1996), these researchers also inferred that, at this point, participants were anticipating the locations of the targets and were performing "memory-based," rather than visually guided, sequences of saccades. However, because the location of the landing position of the saccade was not recorded, it is impossible to know the extent of accuracy of the anticipations over the course of the task.

\section{The Present Study}

In the present study, we used a 10-step spatial sequence, as in the original Nissen and Bullemer (1987) study, and we examined both manual RTs and anticipatory eye movements. The participants were randomly assigned into four groups that differed on type of trial and instructions and were administered eight blocks of trials and three tests of explicit awareness. The experimental design is summarized in Table 1.

Incidental sequence learning (Blocks 1-5). ${ }^{1}$ For the first five blocks, the groups were shown pseudorandom trials only or both sequence and pseudorandom trials. Half the participants in each group pressed buttons in response to the stimuli (push-random and push-sequence groups), whereas the other half were instructed to just look at the stimuli (watch-random and watch-sequence groups). First, we compared manual RTs of the push-sequence and push-random groups. We expected to replicate consis- 
Table 1 Summary of Experimental Design

\begin{tabular}{|c|c|c|c|c|c|c|c|c|}
\hline \multirow[b]{3}{*}{ Group } & \multicolumn{8}{|c|}{ Blocks } \\
\hline & \multicolumn{5}{|c|}{ Incidental Learning } & \multicolumn{2}{|c|}{ Transfer } & \multirow{2}{*}{$\frac{\text { Intentional }}{8}$} \\
\hline & 1 & 2 & 3 & 4 & 5 & 6 & 7 & \\
\hline Watch-random & $\mathrm{RB}$ & $\mathrm{RB}$ & $\mathrm{RB}$ & $\mathrm{RB}$ & $\mathrm{RB}$ & $R B$ & $S B$ & $S B_{2}$ \\
\hline Watch-sequence & $\mathrm{RB}$ & SB & SB & $\mathrm{RB}$ & SB & $R B$ & $S B^{*}$ & $\mathrm{SB}_{2}$ \\
\hline Push-random & $R B$ & $R B$ & $R B$ & $R B$ & $R B$ & $R B$ & $S B$ & $\mathrm{SB}_{2}$ \\
\hline Push-sequence & $R B$ & $S B$ & $S B$ & $R B$ & $S B$ & $R B$ & $S B^{*}$ & $\mathrm{SB}_{2}$ \\
\hline \multicolumn{9}{|c|}{$\begin{array}{l}\text { Note- } \mathrm{RB}=\text { random block, } 100 \text { trials; } \mathrm{SB}=\text { sequence block, } 10 \text { repetitions of 3-2-4- } \\
3-1-4-2-3-4-1 ; S B_{2}=\text { intentional block sequence, } 10 \text { repetitions of 2-1-4-3-1-3-2-1-2- } \\
\text { 4. Response modality: } R B \text { and } S B \text { refer to oculomotor and manual responses. RB and } \\
\text { SB refer to oculomotor response only. }{ }^{*} \text { Following Block } 7 \text {, the watch-sequence and } \\
\text { push-sequence groups were administered the sequence awareness tasks, whereas the } \\
\text { watch-random and push-random groups were not. }\end{array}$} \\
\hline
\end{tabular}

tent SRT findings, in which manual RTs decrease across repeated exposures to sequences and increase for pseudorandom trials. The main goal of the study was to test whether anticipatory eye movements would also reflect sequence learning.

Transfer of learning (Blocks 6-7). All participants were instructed to both look and make manual responses on these blocks. The question here was whether the reduction in manual RTs from Block 6 (pseudorandom) to Block 7 (sequence) would be greater in the watchsequence group than in the watch-random group. This result would provide evidence for transfer of learning from the oculomotor to the manual modality.

Explicit awareness. We assessed awareness by administering three tasks after Block 7 to the groups shown the sequences. Explicit awareness was greater in the participants who simply observed the sequence than in those who made manual responses in Howard et al. (1992) and Heyes and Foster (2002), but not in Willingham (1999, Experiment 1) or Kelly and Burton (2001). These discrepancies were likely due to sequence length and the presence or absence of an initial random block. Although we used a 10-item sequence, as in Howard et al., we also included an initial pseudorandom block to reduce explicit awareness. Thus, we expected explicit awareness to be low, but we tested whether the manual RT and anticipation results would differ after excluding participants who had developed explicit awareness of the sequence.

Intentional sequence learning (Block 8). To explore differences between indices of sequence learning on Blocks 1-5 and indices of intentional sequence learning, we presented an additional block to all participants. Before this trial block, they were told that the stimuli would appear in a certain pattern and were asked to learn it.

\section{METHOD}

\section{Participants}

The participants were 72 students at the University of Minnesota (16 male, 56 female). Their ages ranged from 18.2 to 38.0 years $(M=21.5$ years, $S D=3.3)$. Sixty-one were Caucasian, 2 Hispanic, 1 African-American, and 8 Asian-American. They were paid or earned extra-credit points for psychology classes for their participation. Three additional participants were tested but were excluded because they did not follow directions to look at each stimulus when it appeared.

\section{Apparatus}

\section{Stimulus Presentation}

The participants were seated $69 \mathrm{~cm}$ in front of a VGA color monitor (39 $\mathrm{cm}$ diagonal) on which the stimuli were displayed. Custom software controlled the stimulus display and linked the timing of stimulus presentation with the computer that recorded eye movements. The experiment was conducted in a room with normal ambient illumination.

\section{Manual Response Recording}

A custom response box had four buttons $(1.5 \times 1.5 \mathrm{~cm})$ arranged horizontally with $1.5 \mathrm{~cm}$ between them. The middle and index fingers of the left hand were used for the first and second buttons, and the index and middle fingers of the right hand were used for the third and fourth.

\section{Eye Movement Recording}

Horizontal and vertical coordinates of gaze direction were collected with a video-based eye monitor (ISCAN Eye Tracking Laboratory, Model ETL-400), which has a temporal resolution of $60 \mathrm{~Hz}$ and a spatial resolution of $1^{\circ}$ over the range of visual angles used in this study. A camera with an attached infrared light source to illuminate the pupil was placed in front of the monitor, below eye level, and $40 \mathrm{~cm}$ from the participant. Because the camera automatically compensated for small head movements, no head restraint was used. Custom software merged the eye data with the stimulus presentation and manual response data.

We calibrated eye position for each participant at the beginning of the session by focusing the camera on the participant's left eye and having him/her look at dots in the center and four corners of the screen. These positions were recorded as the targets of eye gaze. This procedure was repeated between testing blocks if necessary due to excessive head movement.

\section{Procedure}

The participants also took part in another set of tasks assessing inhibition during the session. The order of these tasks was counterbalanced as closely as possible. The SRT task took approximately $40 \mathrm{~min}$ to complete. Four boxes $(3.0 \times 3.0 \mathrm{~cm}$, separated by $4.5 \mathrm{~cm})$ were displayed horizontally in the center of the screen. The four boxes together subtended approximately $21^{\circ}$ of visual angle. Boxes were outlined in black against a white background. The stimulus was a colored image of a butterfly. This was chosen in anticipation of a follow-up study with children. The stimulus was shown for $1,000 \mathrm{msec}$, with a $500-\mathrm{msec}$ intertrial interval. Although these 
durations are somewhat longer than in some other SRT studies, we chose them because they would provide enough time to record responses from participants of different ages.

The participants were assigned to one of four groups that differed on response and trial type (see Table 1). Using terminology based on Willingham (1999), we labeled the groups as follows: (1) the push-sequence group $(N=17)$ made manual responses on the first five blocks, and they were shown sequence trials on Blocks 2, 3, and 5 and pseudorandom trials on Blocks 1 and 4; (2) the push-random group $(N=19)$ made manual responses but were shown only pseudorandom trials on these five blocks; (3) the watch-sequence group $(N=18)$ did not respond manually and were shown sequence trials on Blocks 2, 3, and 5; and (4) the watch-random group $(N=18)$ did not respond manually and were shown only pseudorandom trials. Eye movements were recorded for each group. The participants were instructed to look at each stimulus but not to use their peripheral vision. They were instructed to respond as quickly as possible but without making mistakes.

Each block consisted of 100 trials. In the sequence blocks, a repeating 10-item sequence was presented 10 times. This sequence was 3-2-4-3-1-4-2-3-4-1 (based on Beldarrain, Grafman, PascualLeone, \& Garcia-Monco, 1999), with the numbers referring to boxes from left to right. This sequence was chosen because each transition between two consecutive locations (first-order or pairwise transitions) occurred only once during the sequence and because it did not contain any salient fragments, such as 1-2-3-4. In Blocks 1,4 , and 6 , the stimuli were presented in a pseudorandom order that was constrained so the frequency of locations matched that of the sequence trials. In addition, for every 20 trials (1-20, 21-40, etc.) of the pseudorandom block, the frequency of first-order transitions matched that of the sequence block. Because the trials were so highly constrained, differences between the sequence and pseudorandom blocks cannot be explained by the frequency of the locations or the first-order transitions. Instead, they must reflect learning of more complex aspects of the sequence, such as segments of three or more consecutive elements (second-order transitions or higher). The same 100-trial order was used for each pseudorandom block. Block 1 was preceded by 10 practice trials.

Although a 10-item sequence has an unequal distribution of the four stimulus locations, we chose this length rather than a 12-item sequence because we wanted to ensure that learning effects would be found when we attempted this task with children.

\section{Sequence Awareness Tasks}

After Block 7, the participants in the watch- and push-sequence groups completed three tasks to assess their sequence awareness. First, they were asked: "Did you notice anything about the order in which the butterflies appeared?" Regardless of their response, they were informed that the butterflies had sometimes appeared in a repeating sequence. On the recall task, the boxes were displayed on the screen, and the participants were instructed to press the buttons in the order that they thought the butterflies had appeared. They were given $45 \mathrm{sec}$ to make responses, but only the first 20 buttonpresses were analyzed. Because our software could not display images based on buttonpresses, the stimuli did not appear on the screen. In free recall tasks in other SRT studies, the stimuli are usually displayed as the buttons are pressed.

On the prediction task, a stimulus was displayed in one of the boxes, and the participants verbally predicted the box in which they thought the next stimulus would appear. They were told to label the boxes as $1,2,3$, and 4 , from left to right. They did not receive direct feedback about their guesses, but they could use the location of the subsequent stimulus to determine whether they were correct. Twenty trials were administered, consisting of two repetitions of the sequence.

\section{Intentional Learning}

After the seventh block, all participants were shown an additional block of trials (Block 8). They were told that this block would con- tain a new repeating sequence and that the stimulus would be a picture of a crab. All participants responded by looking at the stimuli and making buttonpresses. They were instructed to try to learn the sequence and were told to label the boxes as $1,2,3$, and 4 , but they were not given any information about the length of the sequence. This new sequence (2-1-4-3-1-3-2-1-2-4) was repeated 10 times. After this block, the participants were asked to describe the pattern and what they did to try to learn it. One participant's data on Block 8 were lost due to examiner error.

\section{Dependent Variables}

\section{Manual RTs}

After the eye movement and stimulus data were merged, an algorithm extracted the manual response speed and accuracy. Because the sampling rate of the eye monitor determined the temporal resolution of the merged data file, RTs were accurate to within $16.7 \mathrm{msec}$. Errors or responses prior to stimulus onset were not included in further analyses.

\section{Oculomotor Responses}

Anticipations prior to stimulus onset. This measure estimates participants' correct anticipations of the stimulus locations. A response was counted as an anticipation if (1) the location of the participant's gaze fell within the correct box at the time the stimulus appeared, (2) their gaze had not been located in the box for more than $1,350 \mathrm{msec}$ prior to the onset of the stimulus, and (3) no further saccades were initiated for $1,000 \mathrm{msec}$ after the stimulus onset. The 1,350 -msec cutoff was used to avoid the possibility that a correct anticipation would be confused for an inappropriate response on the previous stimulus. The 1,000-msec limit on further saccades was used to ensure that random eye movements, such as moving the eyes rapidly from side to side, would not be credited as correct anticipations. For an anticipation to be correct, the horizontal location of the gaze had to fall within $20 \mathrm{~mm}\left(1.66^{\circ}\right)$ of the appropriate box for at least two consecutive samples $(33.3 \mathrm{msec})$.

Saccadic response after stimulus onset. A custom program extracted information about poststimulus saccades from the raw data file. To detect saccades, the program first removed blinks. Blinks were defined as (1) the pupil diameter falling below $1.86 \mathrm{~mm}$ or above $5.96 \mathrm{~mm}$, (2) the horizontal or vertical positions of the eye falling outside the limits of the screen, or (3) the diameter of the pupil changing by more than $0.74 \mathrm{~mm}$ over $16.7 \mathrm{msec}$.

A saccade was defined as an eye movement with a velocity between $90^{\circ}$ and $800^{\circ} / \mathrm{sec}$ over at least $33.3 \mathrm{msec}$. Saccadic RT was the time between stimulus onset and the first saccade. Saccades occurring less than $50 \mathrm{msec}$ after a blink were excluded as potential artifacts. Saccadic RTs more than $2 S D$ above the participant's mean RT on that block were excluded as outliers. Saccade position was location of the gaze $66.7 \mathrm{msec}$ after the onset of the saccade. When saccadic RTs were examined as a dependent variable, no differences were detected across groups.

Using these definitions, oculomotor movements (anticipations or saccades) were detected on $87 \%$ of all trials. Blocks on which fewer than 70 oculomotor movements were recorded were examined visually. If low oculomotor response counts appeared to be due to imprecise calibration or loss of eye tracking integrity, the eye movement data were eliminated for all blocks for that participant; however, the manual responses were still included. Of the 72 participants, oculomotor data were excluded for 8: 2 from the watch-random group, 3 from the watch-sequence group, 1 from the push-random group, and 2 from the push-sequence group.

\section{Derived Measures of Sequence Learning}

Two a priori measures were used to indicate sequence learning. The first was the difference between Block 3 and Block 1. This reflected general motor facilitation as well as learning that occurred after 20 repetitions of the sequence. The second indicator was the difference between Block 4 (pseudorandom) and the average of 
Blocks 3 and 5 (sequence). This reflected interference due to random trials after repeated exposure to sequences. These indices were calculated for manual RTs and oculomotor anticipations.

\section{Sequence Awareness Tasks}

Responses to the verbal query were coded as to whether or not the participants spontaneously reported any awareness of a pattern. The scores for the recall task were the longest string of responses that matched any part of the sequence and the number of correct triplets produced. Triplets were three consecutive buttonpresses matching any three consecutive elements in the sequence. For the prediction task, the scores were the longest string of correct responses and the overall number of correct responses out of the 20 trials.

\section{Intentional Sequence Learning}

Manual RTs and oculomotor anticipations were calculated as for Blocks 1-7. The learning score for the verbal report after Block 8 was the longest string of responses matching any part of the sequence.

\section{RESULTS}

\section{Incidental Sequence Learning (Blocks 1-5): Manual Response}

Manual response accuracy across all blocks was $98.4 \%$ $(S D=0.4 \%$, range $=98 \%-100 \%)$. Analyses are based on the mean RT for each participant's correct responses within each block.

We expected that for the push-sequence group, but not for the push-random group, RTs would decrease from Blocks 1 to 3 and increase on Block 4. These planned comparisons were conducted using paired $t$ tests within groups and independent $t$ tests between groups.

Figure 1 illustrates the manual RT results. As predicted, within-group comparisons indicated that for the pushsequence group, the mean RT decrease of $36.1 \mathrm{msec}$
$(S D=33.3)$ from Block 1 to Block 3 was significant $[t(16)=4.47, p<.001]$. However, the decrease of $19.6 \mathrm{msec}(S D=35.2)$ for the push-random group also reached significance $[t(18)=2.43, p=.026]$. A $t$ test on the RT change between Blocks 1 and 3 showed no group difference $(p=.16)$. The reduction in RT for the pushrandom group might have been due to factors such as general motor facilitation, learning the frequency of the locations, or learning first-order transitions between stimuli.

The second learning indicator was the effect of interference on Block 4. As predicted, the difference between Block 4 and the average of Blocks 3 and 5 was significant for the push-sequence group $[t(16)=5.65, p<.001]$, but not the push-random group. This difference was also larger for the push-sequence group than for the pushrandom group $[t(34)=4.42, p<.001]$.

Results support previous SRT studies by showing (1) a decrease in RT when the sequence is first presented and (2) an increase in RT when the pseudorandom block is presented after sequence learning has occurred.

\section{Incidental Sequence Learning (Blocks 1-5): Anticipatory Eye Movements}

Although the participants were not instructed to guess where stimuli would appear, correct anticipations nevertheless occurred on almost a third of the trials across all blocks $(M=31.8, S D=8.1)$. The mean number of anticipations per block was used as the dependent variable.

We first tested whether the groups differed on the basis of whether manual responses were also made. For the groups shown the sequence blocks, a 2 (watch-sequence group vs. push-sequence groups) $\times 5$ (Blocks 1-5) ANOVA showed no group difference $(p=.617)$ or

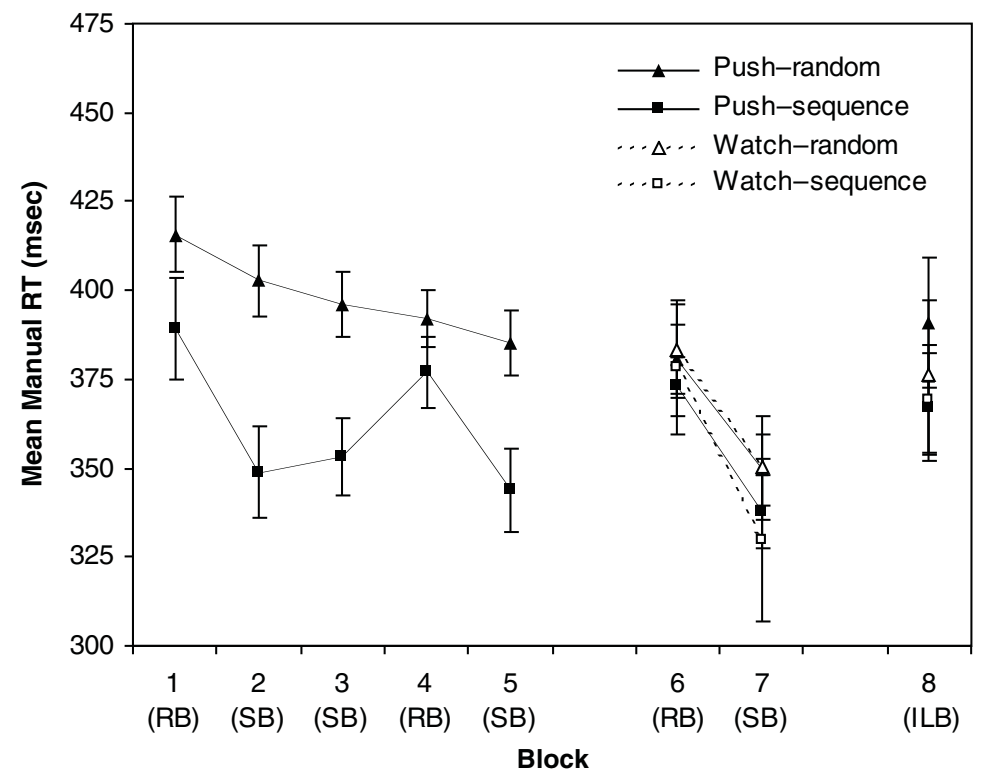

Figure 1. Manual RTs across blocks by group. The watch-sequence and watch-random groups did not make manual responses on Blocks $1-5$, only on Blocks 6-8. RB, random block; SB, sequence block; ILB, intentional learning block. 


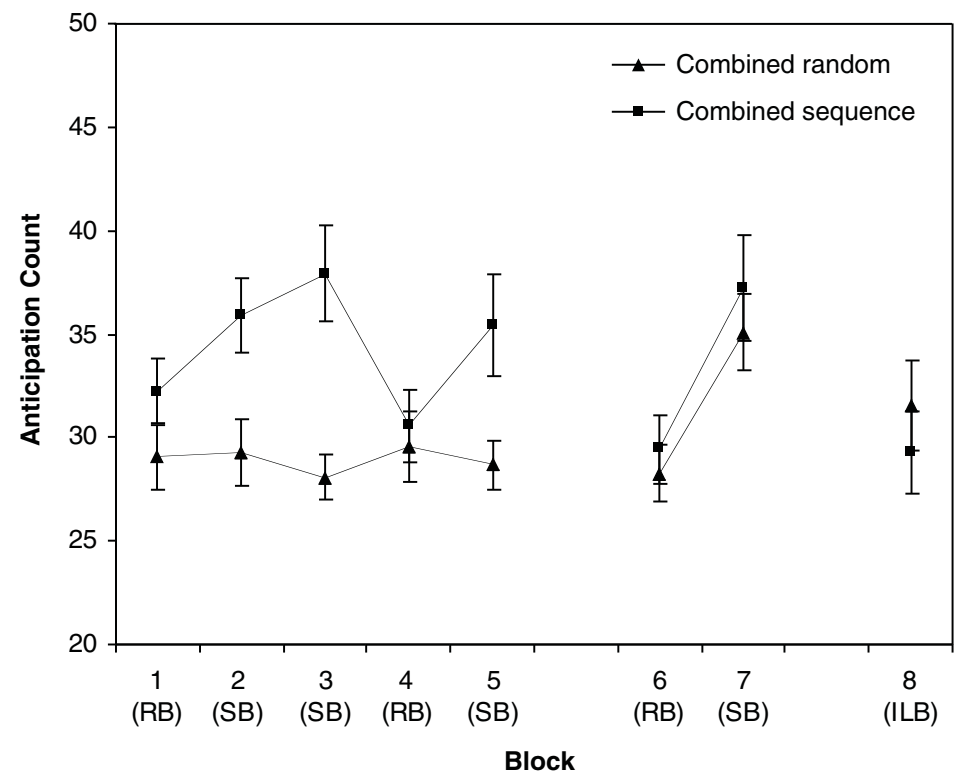

Figure 2. Anticipation counts across blocks by group. Combined sequence and random groups were formed on the basis of analyses of separate groups. $\mathrm{RB}$, random block; SB, sequence block; ILB, intentional learning block.

group $\times$ block interaction $(p=.430)$. These groups were therefore combined into a single sequence group for analysis of anticipations. A parallel analysis for the push- and watch-random groups also showed no group effect ( $p=$ $.491)$ or group $\times$ block interaction $(p=.876)$, so these were combined into a single random group. Figure 2 displays the anticipations made across blocks by these combined groups.

Analyses were conducted on these combined sequence and random groups using the same comparisons as for manual RTs. The increase of $5.7(S D=11.7)$ anticipations between Blocks 1 and 3 was significant for the sequence group [t(29) $=-2.7, p=.012]$, but the decrease of $0.97(S D=8.0)$ anticipations for the random group was not. Between groups, this difference also was significant $[t(62)=-2.69, p=.009]$.

The effect of interference for anticipations was also tested as for manual responses. For the sequence group, the average decrease of $6.11(S D=8.59)$ anticipations in Block 4 was significant $[t(29)=-3.90, p=.001]$, but for the random group, the increase of $1.18(S D=8.58)$ was not. Between the groups, this difference again was significant $[t(62)=-3.39, p=.001]$.

Thus, as shown in Figure 2, the pattern for anticipations and manual RTs was similar, but in the opposite direction. The same pattern was obtained regardless of whether the participants also made manual responses.

\section{Transfer of Learning}

Next, we tested whether learning would transfer from the oculomotor to the manual modality. This was measured for the watch-random and watch-sequence groups, who were making manual responses for the first time on
Block 6 (sequence) and Block 7 (pseudorandom). Although we expected both groups to be faster on Block 7 than on Block 6, we tested whether this reduction in RT would be greater in the watch-sequence participants because of their prior exposure to the sequences. Contrary to predictions, a 2 (watch-sequence group vs. watchrandom group) $\times 2$ (Block 6 vs. Block 7 ) ANOVA showed an effect of block $[F(1,34)=38.7, p<.001]$, but no group effect $(p=.608)$ or interaction $(p=.242)$.

As shown in Figure 1, manual RTs of the watchsequence group $(M=378.3, S D=79.9)$ and the watchrandom group $(M=383.0, S D=54.6)$ were almost identical in Block 6. As expected, RTs for both groups decreased from Block 6 to Block 7. Although the watch-sequence group $(M=329.8, S D=96.7)$ was slightly faster than the watch-random group $(M=350.1, S D=61.7)$ in Block 7, the effect size of the difference was too small to yield a significant result $(d=.25)$. Because the participants in the watch-random group appeared to improve their performance very rapidly in Block 7, we analyzed manual RTs on each set of 20 trials within this block to test for differences in how the groups responded. A 2 (watch-sequence group vs. watch-random group) $\times 5$ (set) ANOVA did not yield any effect of group or set or an interaction (all $p \mathrm{~s}>.4$ ).

\section{Explicit Awareness}

The participants in the watch- and push-sequence groups were first asked whether they had noticed anything about the order in which the stimuli appeared. Overall, 16 of 35 participants in the two groups (46\%) reported some awareness of a pattern. This percentage differed between groups: 12 of $18(67 \%)$ participants in the watchsequence group reported awareness, whereas only 4 of 17 


\begin{tabular}{|c|c|c|c|c|c|}
\hline \multirow[b]{4}{*}{ Task } & \multicolumn{4}{|c|}{$\begin{array}{l}\text { Table } 2 \\
\text { Results for Sequence Awareness Tasks }\end{array}$} & \multirow[b]{4}{*}{ Group Comparison } \\
\hline & & & & & \\
\hline & \multicolumn{2}{|c|}{ Watch-Sequence } & \multicolumn{2}{|c|}{ Push-Sequence } & \\
\hline & $M$ & $S D$ & $M$ & $S D$ & \\
\hline \multicolumn{6}{|l|}{ Recall } \\
\hline Longest correct string* & 6.2 & 1.8 & 4.6 & 1.2 & $t(33)=2.92, p=.006$ \\
\hline No. of correct triplets* & 5.8 & 2.1 & 5.3 & 2.2 & $t(33)=0.80, p=.432$ \\
\hline \multicolumn{6}{|l|}{ Prediction } \\
\hline Longest correct string* & 4.9 & 2.6 & 3.7 & 1.5 & $t(33)=1.73, p=.094$ \\
\hline Total number correct ${ }^{\dagger}$ & 11.8 & 2.5 & 10.1 & 2.8 & $t(33)=1.94, p=.061$ \\
\hline
\end{tabular}

$(24 \%)$ in the push-sequence group did so $\left[\chi^{2}(1)=6.56\right.$, $p=.01]$.

The next tasks examined the extent of this awareness. Table 2 displays these results. For the recall task, the scores were the longest string correct and the number of correct triplets. This produced mixed evidence of group differences. For the longest correct response string, there was a clear advantage for the watch-sequence group, but for the number of correct triplets from the sequence, the difference between groups was not significant. In addition, on the basis of a computer simulation of this task in which 100,000 random iterations of 20 button responses were used to simulate random task performance, it was determined that a string of 7 or more correct was necessary to demonstrate above-chance performance beyond an $\alpha=.05$ level. For the watch-sequence group, 7 of 18 participants (39\%) attained this level; for the push-sequence group, none of the 17 participants did. On the prediction task, both performance measures favored the watchsequence group and showed trends toward significance, although neither reached significance. Results also showed that the participants who stated that they noticed that the stimuli appeared in a pattern did not differ from those who stated that they did not notice a pattern in terms of the longest string of correct responses produced on either the prediction task ( $p=.318)$ or the recall task $(p=.158)$ or in terms of triplets on the recall task $(p=.68)$.

The results reported in previous sections did not change when we reanalyzed the data after excluding the 7 participants from the watch-sequence group whose performance was above chance level on the recall task. That is, a 2 (group) $\times 5$ (block) ANOVA showed no difference in frequency of anticipations in Blocks $1-5$ between the watch-sequence and push-sequence groups $(p=.659)$ and no interaction $(p=.222)$. In the combined sequence group, the frequency of anticipations still declined from Block 1 to Block 3, $[t(22)=2.67, p=.014]$ and showed an interference effect on Block $4[t(22)=2.92, p=.008]$. These effects were greater in the sequence group than in the random group $[t(55)=2.59, p=.012]$. A 2 (watchsequence group vs. watch-random group) $\times 2$ (Block 6 vs. Block 7) ANOVA still showed no interaction for manual RTs $(p=.611)$.

Thus, a majority of the participants were not aware of the sequence. Even those who were aware that a pattern was present did not demonstrate extensive knowledge of what the sequence was, nor did they perform better on the prediction and recall tasks than those who did not have this awareness. The participants who looked at the stimuli but did not respond manually were more likely to gain awareness and to demonstrate more explicit knowledge of the sequence than were the participants who responded manually. However, excluding the 7 participants from the watch-sequence group who demonstrated above-chance performance on the recall task did not change these results.

In addition, there was clear evidence of sequence learning in terms of both the anticipatory eye movements and the manual RTs in the two random groups on Block 7 , after they had been exposed to 600 pseudorandom trials. Unfortunately, we did not assess sequence awareness in these two random groups. However, on the basis of results from the sequence groups, it is unlikely that they would have developed explicit awareness during this block. Yet, their results were very similar to those of the two sequence groups on Block 2.

\section{Intentional Sequence Learning}

To compare intentional sequence learning with sequence learning on the previous blocks, we first examined differences in manual RTs and anticipations between Block 8 and Blocks 2 and 7. Only the push-sequence group was included in the analyses for manual RTs, and the combined sequence group was included in the analyses for anticipations. For these analyses, the mean RT and anticipation count was calculated for each set of 20 trials within the block.

A 3 (Blocks 2, 7, and 8) $\times 5$ (set) ANOVA on manual RTs showed a trend toward a block effect $[F(2,32)=2.72$, $p=.081]$, a set effect $[F(4,64)=3.88, p=.007]$, and an interaction $[F(8,128)=5.19, p<.001]$. We followed up these results with within-block analyses of set effects. As shown in Figure 3, there was a large set effect in Block 8 $[F(4,64)=7.16, p<.001]$, but not in Block 2 or Block 7 $(p s>.201)$. The linear trend in Block 8 was significant $[F(1,16)=11.49, p=.004]$ and linearity accounted for $92 \%$ of the variance in the function relating RT to set.

Anticipation results are displayed in Figure 4. A 3 (Blocks 2, 7, and 8) $\times 5$ (set) ANOVA on anticipations in the combined sequence group showed a block effect $[F(2,60)=5.41, p=.007]$ and a set effect $[F(4,120)=$ $33.54, p<.001]$, but no interaction. The linear trend for set showed that anticipations increased linearly with each 


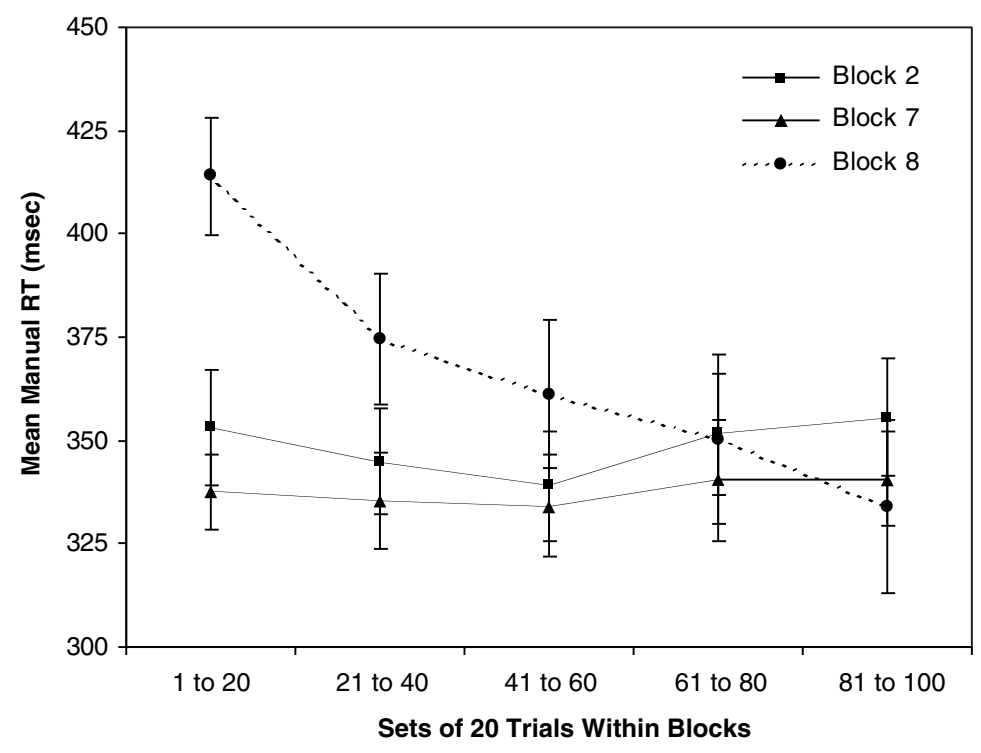

Figure 3. Manual RTs for push-sequence group within incidental and intentional blocks by set of 20 trials.

set within blocks $[F(1,30)=68.78, p<.001]$, with linearity accounting for $91 \%$ of the variance.

Thus, manual RTs remained stable across the five sets of 20 trials within Blocks 2 and 7 but declined linearly from the first to the last 20 trials in the intentional block. In contrast, anticipations increased linearly within all three blocks.

Next, we examined correlations between anticipations and manual RTs across all participants in the last three blocks. Faster manual RTs were correlated with more anticipations in Block $7[r(64)=-.42, p<.001]$ and Block $8[r(63)=-.25, p=.047]$, but not in Block 6 $(p=.14)$. The relation between anticipations and manual RTs in Block 7 remained significant even after the participants who became explicitly aware were excluded $[r(57)=-.29, p=.032]$.

To determine whether anticipations were related to explicit awareness and recall of the sequence, we conducted a 2 (explicitly aware or not) $\times 5$ (Blocks $2,3,5$, and 7) ANOVA on anticipations during the sequence blocks in the watch-sequence and push-sequence groups. Results showed no difference between participants with and without explicit awareness $(p=.866)$ and no interaction between awareness and block $(p=.722)$. Within these two sequence groups, there was also no correlation between anticipations in Block 7 and longest string of correct responses or triplets on the recall or prediction tasks ( $p \mathrm{~s}>$ $.21)$. In contrast, in Block 8 , number of anticipations was positively related to learning score on the verbal recall task across all participants $[r(62)=.31, p=.014]$.

\section{DISCUSSION}

\section{Incidental Sequence Learning (Blocks 1-5)}

Manual RTs replicated robust learning effects found in previous SRT studies. Anticipatory eye movements in both the watch- and push-sequence groups closely paralleled the pattern of manual RTs, but in the opposite direction. These results extend the results of previous studies showing anticipatory eye movements in monkeys (Miyashita et al., 1996) and humans during spatial sequence learning (Kawashima et al., 1998). This evidence of SRT learning in both manual and oculomotor modalities was found, despite the fact that the pseudorandom trials had the same overall frequency and first-order transitional properties as the sequence trials, a constraint that made it less likely to find differences. In addition, anticipations increased quickly in the two random groups on the first block in which they were exposed to the sequence (Block 7), replicating results in sequence groups on Block 2. Taken together, these results clearly show that anticipatory eye movements reflect sequence learning and that they occur equally frequently whether or not participants make concurrent manual responses.

\section{Transfer of Learning}

Although manual RTs of the watch-sequence and watch-random groups were virtually identical in Block 6 and the watch-sequence group was slightly faster on Block 7, the difference did not reach significance. This failure to find transfer appeared to be due to extremely fast learning in the watch-random group, who had been exposed to only 600 pseudorandom trials up to that point. The fact that sequence learning occurred so quickly implies that observational studies in which participants were initially provided with manual practice on relatively short sequences (Heyes \& Foster, 2002, Experiments 1 and 2; Howard et al., 1992, Experiment 1) cannot have measured "pure perceptual learning." However, because the temporal resolution of the manual RTs was $17 \mathrm{msec}$, we may have been unable to detect small differences in RTs. Thus, transfer of learning needs to be assessed further in designs 


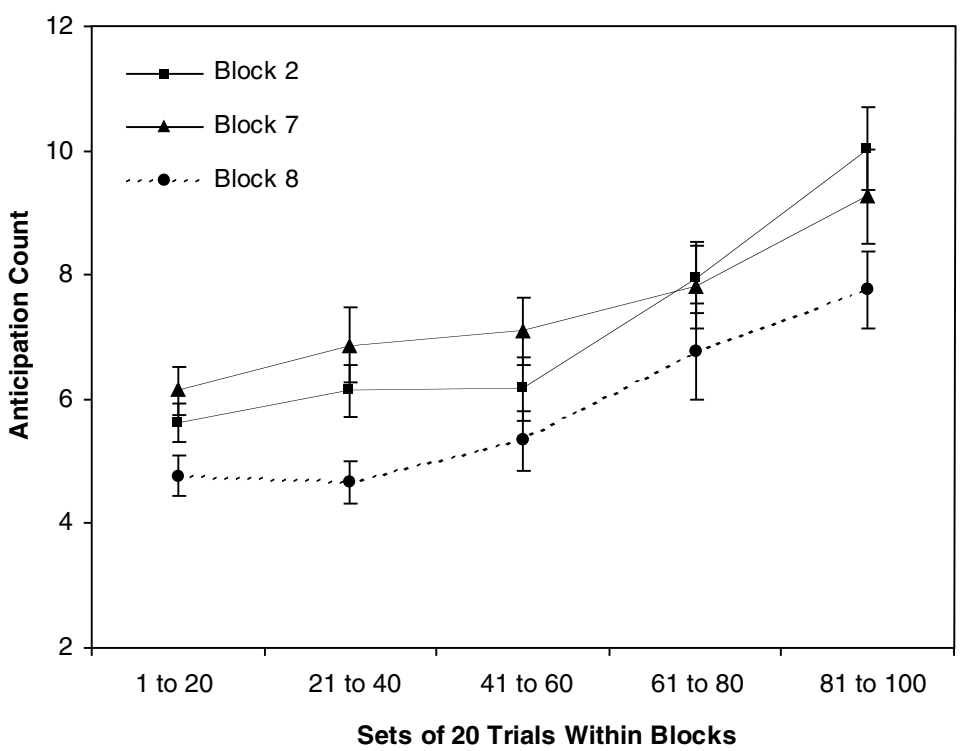

Figure 4. Anticipations for push-sequence group within incidental and intentional blocks by set of 20 trials.

that are more sensitive to small effects or with more difficult sequences that may not be learned as quickly.

\section{Explicit Awareness}

Although almost half the participants expressed some awareness of a pattern, few were able to accurately reproduce any chunks from the sequence. In addition, those who professed awareness of a pattern did not perform better on the recall or prediction tasks than those who professed no awareness.

As in Heyes and Foster (2002) and Howard et al. (1992), the participants who only looked at the stimuli developed greater explicit awareness than did the participants who both looked and made manual responses. This difference probably occurred because these participants were more likely to think about stimulus locations or the purpose of the task when they were not responding manually. However, anticipations were not more frequent in the participants who had developed explicit awareness, and results did not change when the participants who performed above chance on recall were excluded from the analyses. Thus, making manual responses did not affect frequency of anticipations, but reduced explicit awareness. In future studies examining anticipatory eye movements, it would be advisable to have participants make both eye and hand movements.

\section{Intentional Sequence Learning}

Manual RTs were stable throughout the first and last incidental sequence blocks (Blocks 2 and 7) but decreased linearly with each set of 20 trials within the intentional block (Block 8). In contrast, anticipations increased linearly within blocks, regardless of condition. The participants who made more anticipations also had faster manual responses in Blocks 7 and 8, but not in Block 6 (pseudorandom). In addition, the participants who made more anticipations in Block 8 (intentional) also verbally recalled more of the sequence.

\section{What Do Anticipatory Eye Movements Reflect?}

Eye movements and shifts of visuospatial attention are normally coupled, and it is plausible to assume that the anticipations we observed were manifestations of shifts of visuospatial attention to likely stimulus locations. In addition, these shifts of attention and eye movements appeared to help speed up responses in both the incidental block and the intentional block (cf. Posner, 1980). The only difference between anticipations in the incidental and intentional blocks was that anticipations were positively related to verbal recall of the sequence in the intentional block, but not in the incidental block. Thus, these anticipations were probably reflecting a conscious strategy and explicit awareness in the intentional block, but not in the incidental block.

Less obvious, but equally interesting, is the fact that the anticipations occurred spontaneously from the beginning, whether or not there was a sequence, and continued to occur at a stable rate throughout the six pseudorandom blocks in the watch- and push-random groups. Thus, rather than reflecting processes specific to sequence learning, these anticipations may have been reflecting a more fundamental search for regularities in the environment that occurs constantly, whether or not there are regularities. These results are consistent with the findings of a study in which participants were shown 1,800 stimuli one at a time and instructed to press one of two buttons depending on the stimulus (Huettel, Mack, \& McCarthy, 2002). The order of the stimuli was random, and partici- 
pants were informed of this beforehand. The researchers later extracted patterns that occurred by chance (2-8 consecutive runs of alternating or repeating stimuli) and found increased and differential brain activity in response to violations of these "local" patterns. The researchers concluded that "recognition of patterns is an [automatic] obligatory, dynamic process that includes the extraction of local structure from even random sequences" (p. 489). Similar sensitivity to local statistical structure has been found in pigeons on a variant of the SRT task (Froehlich, Herbranson, Loper, Wood, \& Shimp, 2004). In this study, the pigeons were faster to respond when the location of the stimulus could be predicted from the locations of the previous one or two stimuli, indicating that anticipations of forthcoming stimuli based on local patterns does not necessarily reflect high-level cognitive strategies.

In conclusion, we hypothesize that the sequence of events on the task in this study may be as follows: Before the onset of the stimulus, participants shift both visuospatial attention and direction of gaze to a likely stimulus location. These shifts occur whether or not there is a sequence and whether or not a manual response is required; they may be guided by conscious hypothesistesting strategies, as in the intentional block, but need not be. The stimulus then appears, and participants register the discrepancy between their prediction and the actual location. If the prediction was accurate and a manual response is required, the manual response is fast. If it was inaccurate, attention is shifted (overtly or covertly) to the new location. Thus, participants are constantly anticipating the stimuli from the beginning, and it is the accuracy of these anticipations that appears to be improving during sequence learning (and possibly during the pseudorandom blocks as well).

\section{Suggestions for Future Research}

One of the limitations of this study is that all participants were instructed to look at the stimuli. Therefore, it would be informative to compare these results with those of a condition in which participants were not provided with any instructions regarding where to look.

It would also be informative to compare the results of this study with those of a condition in which the stimuli were placed as far apart as possible, but in which participants were instructed to rely on peripheral vision alone and not to make eye movements. This comparison would reveal whether eye movements are simply epiphenomena of covert shifts of attention or whether they play an instrumental role in sequence learning. To our knowledge, no study of spatial sequence learning has restricted eye movements. Two studies used stimuli that were spaced closely (Helmuth et al., 2000; Willingham et al., 1989, Experiment 3), which would minimize the need to make eye movements. Spatial sequence learning was not observed in these studies. These results were interpreted as indicating that what is learned is not shifts of attention but a sequence of stimulus-response mappings (Willingham et al., 1989) or as indicating that even if the spatial loca- tions may have been learned, the benefits of this learning were too small to have been detected in manual RTs (Helmuth et al., 2000; Mayr, 1996). Remillard (2003) investigated the effect of distance on sequence learning, using closely spaced stimuli in a series of four experiments. Results showed that participants could learn the sequence of locations, regardless of how closely spaced the stimuli were, leading Remillard to conclude that "eye movements were not necessary for learning" (p. 592). It still remains an open question, however, whether eye movements play a facilitatory role for learning spatial sequences of stimuli placed farther apart.

It is also important to consider the role of temporal factors (e.g., Buchner \& Steffens, 2001; Lee, 2000; Shin \& Ivry, 2002). Froehlich et al. (2004), for instance, report that there are optimal temporal parameters for response-stimulus intervals that are similar across pigeons and humans and that presenting stimuli at a faster or slower rate hinders learning of local statistical structure. Charting the relations between efficiency of sequence learning versus spatial and temporal parameters of the stimuli may reveal a great deal about the nature of visuospatial anticipatory mechanisms and the role of working memory in sequence learning.

In this study, the stimulus locations were identical to the locations that the participants had to look at or respond to manually. We found that anticipations were similar between the participants who made manual responses and those who did not. Thus, it is unlikely that these eye movements were anticipating the location to which a manual response had to be made. However, further research is needed to decide whether the participants were anticipating the locations to which they needed to make an oculomotor movement or the locations of the stimuli.

A broader question pertains to the nature of sequence learning in spatial versus nonspatial modalities. On the one hand, sequence learning may involve anticipatory shifts of visuospatial attention for spatial sequences but other (Mayr, 1996), perhaps even less effective (Koch \& Hoffman, 2000), mechanisms for nonspatial sequences. On the other hand, there may be anticipatory mechanisms that operate regardless of the stimulus modality.

\section{Implications for Research on Sequence Learning}

First, the results provide information on what participants are actually looking at when they are learning a spatial sequence. In previous studies, participants were not given any specific instructions about whether or not they should look at the stimuli, and they were simply instructed to "watch" or "observe." In the present study, we explicitly instructed the participants to look at the stimuli. The data show that, under these conditions, the participants were in fact making many oculomotor movements. These results cast doubt on perceptual or S-S learning theories that claim that sequence learning occurs during observation in a "purely perceptual" manner.

Second, the results not only show that the participants were making eye movements during sequence learning but provide clues as to the function of these movements. 
Although the participants were not instructed to try to anticipate where the stimulus would appear, they nevertheless made many spontaneous anticipatory eye movements. These results provide support to the hypothesis that sequence learning involves learning to anticipate the location of the upcoming stimulus (Hoffman et al., 2003; Nissen \& Bullemer, 1987; Remillard, 2003; Stadler, 1989). They also extend to the results of previous studies showing that humans and monkeys direct their eye movements in an anticipatory manner in many other tasks. After reviewing research on anticipatory eye movements in sports, driving, and reading, Land and Furneaux (1997) concluded that eye movements are oriented toward the future, so as to prepare for forthcoming action:

Like an advance patrol, the eye movement system is out gathering useful intelligence for the performance of whatever motor strategy is at hand. Thus, in contrast to the impression one gets from laboratory studies where eye movements are treated as "responses" to "stimuli," most eye movements in the real world are proactive in nature, not reactive. (pp. 1231-1232)

In discussions of eye movements on the SRT task, eye movements are often referred to as responses (e.g., Mayr, 1996; Tubau \& López-Moliner, 2004). The present results challenge this view. The anticipatory eye movements we observed during the SRT task should not be thought of as responses but as advance patrols that occur even when accurate predictions are not necessary and have no obvious reward value, no conscious problem-solving strategies are used, and no other motor system is necessary for responding.

Third, by demonstrating that anticipatory eye movements occur from the beginning of the SRT task in both the random block and the sequence block, the data point to the need to extend current theories based solely on associationist principles. That is, it may not be completely accurate to view sequence learning as a passive process of building simple associations on a blank slate between stimuli and responses, between consecutive responses, or between responses and their effects. Instead, sequence learning appears to be built on a foundation of constant anticipatory activity, which may help explain why it occurs so rapidly.

Finally, these findings highlight the fact that different kinds of attentional processes may be involved in the SRT task. As Nissen and Bullemer (1987) noted, "the relation between attention and memory is central to theories of attention and theories of memory" (p. 1). The kind of attention they were interested in is the kind that is rooted in resource theories, measured on dual-task paradigms and is relevant to the distinction between implicit and explicit learning. Discussions of the role of attention in sequence learning have been framed mostly in these terms since then. However, recent research has begun to challenge the primacy of the implicit-explicit distinction in understanding sequence learning (e.g., Keele, Ivry, Mayr, Hazeltine, \& Heuer, 2003; Wilkinson \& Shanks, 2004). We would like to add to this discussion the reminder that there are varieties of attention that may be separable from each other (reviewed in Karatekin, 2001). The kind of attention we focused on in this study — visuospatial attention-does not need to be tied to high-level cognitive strategies or explicit awareness and is more closely intertwined with motor planning. Thus, research on anticipatory shifts of visuospatial attention and eye movements may be as informative as previous studies of attention in illuminating mechanisms of sequence learning in healthy young adults, as well as in developmental and clinical populations.

\section{REFERENCES}

Beldarrain, M. G., Grafman, J., Pascual-Leone, A., \& GarciaMonco, J. C. (1999). Procedural learning is impaired in patients with prefrontal lesions. Neurology, 52, 1853-1860.

Buchner, A., \& Steffens, M. C. (2001). Simultaneous learning of different regularities in sequence learning tasks: Limits and characteristics. Psychological Research, 65, 71-80.

Froehlich, A. L., Herbranson, W. T., Loper, J. D., Wood, D. M., \& SHImP, C. P. (2004). Anticipating by pigeons depends on local statistical information in a serial response time task. Journal of Experimental Psychology: General, 133, 31-45.

Grafton, S. T., Hazeltine, E., \& Ivry, R. B. (1998). Abstract and effector-specific representations of motor sequences identified with PET. Journal of Neuroscience, 18, 9420-9428.

Helmuth, L. L., Mayr, U., \& Daum, I. (2000). Sequence learning in Parkinson's disease: A comparison of spatial-attention and numberresponse sequences. Neuropsychologia, 38, 1443-1451.

Heyes, C. M., \& Foster, C. L. (2002). Motor learning by observation: Evidence from a serial reaction time task. Quarterly Journal of Experimental Psychology, 55A, 593-607.

Hoffman, J., Martin, C., \& Schilling, A. (2003). Unique transitions between stimuli and responses in SRT tasks: Evidence for the primacy of response predictions. Psychological Research, 67, 160-173.

Howard, J. H., JR., MutTer, S. A., \& Howard, D. V. (1992). Serial pattern learning by event observation. Journal of Experimental Psychology: Learning, Memory, \& Cognition, 18, 1029-1039.

Huettel, S. A., Mack, P. B., \& McCarthy, G. (2002). Perceiving patterns in random series: Dynamic processing of sequence in prefrontal cortex. Nature Neuroscience, 5, 485-490.

KarateKIN, C. (2001). Developmental disorders of attention. In C. A. Nelson \& M. Luciana (Eds.), Handbook of developmental cognitive neuroscience (pp. 561-576). Cambridge, MA: MIT Press

Kawashima, R., Tanji, J., Okada, K., Sugiura, M., Sato, K., Kinomura, S., ET AL. (1998). Oculomotor sequence learning: A positron emission tomography study. Experimental Brain Research, 122, $1-8$.

Keele, S. W., Ivry, R., Mayr, U., Hazeltine, E., \& Heuer, H. (2003). The cognitive and neural architecture of sequence representation. Psychological Review, 110, 316-339.

Keele, S. W., Jennings, P., Jones, S., Caulton, D., \& Cohen, A. (1995). On the modularity of sequence representation. Journal of Motor Behavior, 27, 17-30.

Kelly, S. W., \& Burton, A. M. (2001). Learning complex sequences: No role for observation? Psychological Research, 65, 15-23.

Koch, I., \& Hoffman, J. (2000). Patterns, chunks, and hierarchies in serial reaction-time tasks. Psychological Research, 63, 22-35.

LAND, M. F., \& Furneaux, S. (1997). The knowledge base of the oculomotor system. Philosophical Transactions of the Royal Society of London: Series B, 352, 1231-1239.

LEE, D. (2000). Learning of spatial and temporal patterns in sequential hand movements. Cognitive Brain Research, 9, 35-39.

MAYR, U. (1996). Spatial attention and implicit sequence learning: Evidence for independent learning of spatial and nonspatial sequences. Journal of Experimental Psychology: Learning, Memory, \& Cognition, 22, 350-364.

Miyashita, K., Rand, M. K., Miyachi, S., \& Hikosaka, O. (1996). Anticipatory saccades in sequential procedural learning in monkeys. Journal of Neurophysiology, 76, 1361-1366. 
Nattkemper, D., \& PrinZ, W. (1997). Stimulus and response anticipation in a serial reaction task. Psychological Research, 60, 98-112.

Nissen, M. J., \& Bullemer, P. (1987). Attentional requirements of learning: Evidence from performance measures. Cognitive Psychology, 19, 1-32.

Posner, M. I. (1980). Orienting of attention. Quarterly Journal of Experimental Psychology, 32, 3-25.

Posner, M. I., \& Rothbart, M. K. (1992). Attentional mechanisms and conscious experience. In A. D. Milner \& M. D. Rugg (Eds.), The neuropsychology of consciousness (pp. 91-111). San Diego: Academic Press.

Remillard, G. (2003). Pure perceptual-based sequence learning. Journal of Experimental Psychology: Learning, Memory, \& Cognition, 29, 581-597.

Rüsseler, J., Hennighausen, E., Münte, T. F., \& Rösler, F. (2003). Differences in incidental and intentional learning of sensorimotor sequences as revealed by event-related brain potentials. Cognitive Brain Research, 15, 116-126.

Schvaneveldt, R. W., \& Gomez, R. L. (1998). Attention and probabilistic sequence learning. Psychological Research, 61, 175-190.

Seger, C. A. (1997). Two forms of sequential implicit learning. Consciousness \& Cognition, 6, 108-131.

Shin, J. C., \& IvrY, R. B. (2002). Concurrent learning of temporal and spatial sequences. Journal of Experimental Psychology: Learning, Memory, \& Cognition, 28, 445-457.

Stadler, M. A. (1989). On learning complex procedural knowledge. Journal of Experimental Psychology: Learning, Memory, \& Cognition, 15, 1061-1069.

Thomas, K. M., \& Nelson, C. A. (2001). Serial reaction time learning in preschool- and school-age children. Journal of Experimental Child Psychology, 79, 364-387.

Tubau, E., \& LóPez-Moliner, J. (2004). Spatial interference and response control in sequence learning: The role of explicit knowledge. Psychological Research, 68, 55-63.
Wilkinson, L., \& Shanks, D. R. (2004). Intentional control and implicit sequence learning. Journal of Experimental Psychology: Learning, Memory, \& Cognition, 30, 354-369.

Willingham, D. B. (1999). Implicit motor sequence learning is not purely perceptual. Memory \& Cognition, 27, 561-572.

Willingham, D. B., Nissen, M. J., \& Bullemer, P. (1989). On the development of procedural knowledge. Journal of Experimental Psychology: Learning, Memory, \& Cognition, 19, 1424-1430.

ZiESSLER, M. (1994). The impact of motor responses on serial-pattern learning. Psychological Research, 57, 30-41.

ZiESSLER, M. (1998). Response-effect learning as a major component of implicit serial learning. Journal of Experimental Psychology: Learning, Memory, \& Cognition, 24, 962-978.

ZieSSLER, M., \& NATTKEMPER, D. (2001). Learning of event sequences is based on response-effect learning: Further evidence from a serial reaction task. Journal of Experimental Psychology: Learning, Memory, \& Cognition, 27, 595-613.

ZiRngibl, C., \& KoCH, I. (2002). The impact of response mode on implicit and explicit sequence learning. Experimental Psychology, 49, 153-162.

\section{NOTE}

1. We decided to label the initial blocks as incidental (as in Rüsseler, Hennighausen, Münte, \& Rösler, 2003) rather than as implicit. Although mechanisms of sequence learning are likely to be predominantly implicit in these blocks, the term incidental may be more appropriate, since it does not convey any assumptions regarding whether learning was purely implicit or whether it may have been "contaminated" by explicit awareness.

(Manuscript received May 18, 2003; revision accepted for publication February 3, 2005.) 